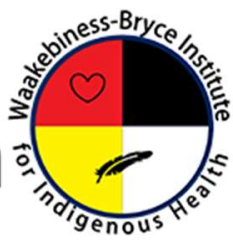

Dalla Lana

School of Public Health

Volume 14

Issue 1. Physical Activity and Cultural Safety

Article 2

DOI 10.32799/ijih.v14i1.31967

May 2019

\title{
Evaluation of the Indigenous Relationship and Cultural Safety Courses among a sample of Indigenous Services Canada nurses
}

Michelle Rand

Indigenous Cancer Control Unit, Cancer Care Ontario

michelle.rand@cancercare.on.ca

Amanda J. Sheppard

Indigenous Cancer Control Unit, Cancer Care Ontario

Dalla Lana School of Public Health, University of Toronto

amanda.sheppard@cancercare.on.ca

Sehar Jamal

Indigenous Cancer Control Unit, Cancer Care Ontario

sehar.jamal@cancercare.on.ca

Alethea Kewayosh

Indigenous Cancer Control Unit, Cancer Care Ontario,

alethea.kewayosh@cancercare.on.ca

Angela Mashford-Pringle, $\mathrm{PhD}$

Associate Director, Waakebiness-Bryce Institute for Indigenous Health, University of Toronto

angela.mashford.pringle@utoronto.ca

Follow the International Journal of Indigenous Health at:

https://jps.library.utoronto.ca/index.php/ijih/index

\section{Recommended Citation}

Rand, M., Sheppard, A., Jamal, S., Mashford-Pringle, A. (2019). Evaluation of the Indigenous Relationship and Cultural Safety Courses among a sample of Indigenous Services Canada nurses. IJIH14(1), 29-41. DOI

10.32799/ijih.v14i1.31967 


\title{
Evaluation of the Indigenous Relationship and Cultural Safety Courses among a sample of Indigenous Services Canada nurses
}

\begin{abstract}
In 2015, Cancer Care Ontario (CCO) launched the Indigenous Relationship and Cultural Safety (IRCS) courses, which support the importance for healthcare professionals to understand and apply First Nations, Inuit, and Métis (FNIM) cultural safety to provide effective person-centred care. The courses address a key recommendation from the Truth and Reconciliation Commission of Canada report, to provide skills-based training in cultural competency, conflict resolution, human rights and anti-racism. The objective of the evaluation was to validate the tool, with a sample of nurses, to assess: if the delivery mechanism is appropriate and feasible; if participants acquire an increased knowledge of the courses' contents; and if positive change in how healthcare practice is delivered is perceived to have resulted. The IRCS courses have been mandated for Indigenous Services Canada (ISC) nurses who service mostly on reserve communities. The evaluation consisted of an anonymous and voluntary survey and a focus group that were conducted at a regional meeting. The responses from the surveys were gathered in an excel spreadsheet for analyses and the focus group data were analyzed for key themes. All the nurses in attendance completed the survey $(n=22)$ and a portion participated in the focus group $(n=8)$. Our evaluation demonstrated that free, online, module formatted courses were appropriate and relevant for ISC nurses (81\%); the courses increased the knowledge about FNIM people (72\%); and the nurses have/will apply what they learned in their practice $(82 \%)$. There has been an increasing movement for regions and organizations in Canada to complete cultural competency training. Our evaluation demonstrated that the IRCS courses were successful at meeting learning objectives.
\end{abstract}

\section{Keywords}

Indigenous, First Nations people, cultural competency, cultural safety, nurse, eLearning education, evaluation.

\section{Creative Commons Licence}

(c) (1)

This work is licensed under a Creative Commons Attribution-Noncommercial-No Derivative Works 4.0 License. 


\section{Introduction}

First Nations, Inuit and Métis (FNIM) people bear a disproportionately high cancer burden and face a number of health disparities, barriers and gaps to health services (Cancer Care Ontario, 2015). One barrier for FNIM people receiving adequate healthcare is the experience of culturally insensitive healthcare including racism and discrimination. Indigenous people may experience racism in the healthcare system so extensive that prior consideration is given or there is avoidance of accessing the healthcare system altogether (Allan \& Smylie, 2015).

Conversely, people who experience culturally safe healthcare are more likely to: access care

earlier, feel more comfort and control while receiving care, share details about their health concerns and preferences, and return for follow up visits and follow treatment plans recommended by healthcare providers (National Collaborating Centre for Aboriginal Health, 2014). People also feel more in control of their health when engaging with a healthcare provider that empowers them to make shared decisions about their care (Jull et al., 2015). In short, cultural safety is a critical component for improving patient experiences and outcomes.

Another barrier for FNIM people receiving culturally appropriate healthcare are the Canadian federal government policies that are in place, specifically policies related to how healthcare is delivered in First Nations communities. Healthcare is provincially legislated and the Canadian provinces receive federal transfer payments under the Canada Health Act to address healthcare related items such as insurance, billing payments, and healthcare professionals (Canada Health Act, 1985). Given that the Indian Act is also currently in place, registered First Nation people are the legal responsibility of the federal government and as such, the delivery of healthcare is the federal responsibility (Indian Act, 1985). The Indian Act and the policy on how healthcare is delivered is based on assimilation and does not allow those under the Act to determine how services should be delivered in their own communities. This in turn, forces Indigenous communities to navigate both a western healthcare system often foreign to them and a traditional system where health is addressed in a holistic way (Mashford-Pringle, 2011). Many Canadians are only starting to understand the complexities of the discriminatory policies for FNIM people and the impacts these policies have had and continue to have on their health and wellbeing. An impact can be made on how policies and services are developed and implemented at institutions, by creating an understanding of cultural safety through educational opportunities (Brascoupé \& Waters, 2009).

In 2015, Cancer Care Ontario (CCO) launched the Indigenous Relationship and Cultural Safety (IRCS) courses. With the release of the Truth and Reconciliation Commission (TRC) of Canada report, the courses address a key recommendation, to provide skills-based training in cultural competency, conflict resolution, human rights, and anti-racism (Truth and Reconciliation Commission of Canada, 2015). The Joint Ontario Indigenous Cancer Committee (JOICC), which is an advisory group to the Indigenous Cancer Control Unit at CCO and consists of First Nations, Inuit, Métis and urban Indigenous 
organizations, played an integral role in the creation of the IRCS courses. JOICC was the group to first suggest that $\mathrm{CCO}$ update the once paper-based cultural competency training modules and move the content to an online format to increase audience access and allow for increased interactivity. The members advised on specific content in their area of expertise and helped to inform the different topics that should be included in the courses.

The 13 IRCS courses are free of charge and although the original target audience were healthcare providers, the courses are open to anyone. The IRCS courses are stand alone modules, do not need to be completed in any particular order and take approximately one and a half hours to complete the interactivity guides, course content, quiz questions and the feedback survey. The courses cover a variety of topics including:

1. First Nations, Inuit and Métis Culture, Colonization and the Determinants of Health

2. Aboriginal History and Political Governance

3. The Need for Cultural Competence in Healthcare

4. Current Array of Aboriginal Health Services

5. CCO - Aboriginal Cancer Strategies and Ontario Renal Network

6. Indigenous Knowledge and Traditional Health

7. Aboriginal Community Health Services

8. The Health Landscape of First Nations, Inuit and Métis People

9. Cancer and Renal Issues and Challenges

10. Truth and Reconciliation Commission of Canada (TRC) and the United Nations Declaration on the Rights of Indigenous Peoples (UNDRIP)

11. Health Literacy - Indigenous Perspectives on Health and Well-Being

12. Chronic Disease Prevention for First Nations, Inuit and Métis People

13. Pediatric Oncology

After review of the online enrollment data (2015-2018), the majority of those who are enrolled or have completed one or more courses identify themselves as registered nurses, followed by the categories of 'other health professionals', 'students' and 'medical doctors' (to name a few). Given that the majority of those on the eLearning site who are enrolled are nurses and the original target audience were healthcare providers, it is valuable to understand the perspective from nurses to determine if the content of the IRCS courses is beneficial to them. This current work is the first phase of a large scale evaluation of the IRCS courses, which will validate the IRCS courses as a tool to determine if change is created in healthcare providers' perceptions of how culturally appropriate care is delivered.

In this first phase of the evaluation of the IRCS courses, we wanted to validate the tool with a group of nurses that work primarily with FNIM people and that have completed the IRCS courses as their cultural competency training. Given that the Indigenous Services Canada (ISC) First Nations and Inuit Health Branch (FNIHB) Ontario region has nurses in the Sioux Lookout Zone area that only 
provide healthcare to First Nations communities in Northwestern Ontario, this was an ideal group to evaluate. Furthermore, in the fall of 2017, through a phased approach, FNIHB made the IRCS courses mandatory for their nurses over the course of a year. Through a collaborative effort, we were able to gain insight on the IRCS courses from the perspective of the FNIHB nurses.

\section{Methods}

There were two parts to this phase of evaluating the IRCS courses, the first of which was a survey. The objective of the survey was to gather contextual and demographic information of the nurses and high-level feedback on their experience and perception of the IRCS courses as a training method for cultural competency. The second part of the data collection was through focus groups, with an objective to further understand how the IRCS courses impact the nurses' understanding of cultural competency and how the tool/training will be applied to their practice.

\section{Participants}

Both the survey and the focus groups included the involvement of the FNIHB Sioux Lookout Zone nurses however, participation was voluntary. Given the vast geographic distances it would take to interview each of the nurses individually, we leveraged a workshop held by FNIHB that brought all of the Sioux Lookout Zone Nurses in Charge together. Nurses in Charge (referred to as 'nurses' from hereon in) are defined by the Government of Canada as those who have the same responsibilities as

community health nurses, in addition to other roles such as, supervising station nurses and support staff, providing support to community health representatives and liaising with local band administration and community organizations (Government of Canada, 2018). By using this meeting to conduct the research, it allowed us to engage with all of the nurses for a survey and those who wanted to participate in a focus group were able to do so as well.

\section{Survey}

The survey tool was designed through a collaborative process, leveraging knowledge from the various partners. There were 26 questions in total which covered topics including: demographics, professional experience, community characteristics where they work, previous and current knowledge of FNIM people, and characteristics of the IRCS courses. The possible responses were all multiple choices and included yes/no, content specific, and Likert scale answers.

The survey was conducted with the nurses who agreed to participate in the research project. We offered no compensation. A consent agreement was first distributed for the nurses to sign and then those who completed the agreement were given the anonymous paper survey. The results of the survey were then gathered and coded in an excel spreadsheet for analysis. Frequency distributions showed the percentage of observations for each question in the survey. 


\section{Focus Groups}

The focus group supplemented the information gathered from the survey and allowed for greater exploration of the high-level feedback that was provided in part one of the research. The focus group was conducted in a one-hour session with the nurses who chose to participate. The nurses who participated in the focus group had already participated in the survey. No demographic or identifying information was collected in the focus groups. During the focus group, two external staff captured the focus group discussion with written notes; the discussion was not recorded. Each note taker independently analyzed the data for key themes and categorized the content based on the most relevant theme.

\section{Relationship}

Ethical approval was obtained from the University of Toronto Research Ethics Board. Engagement with the Sioux Lookout Zone nurses was done through collaborative efforts led by CCO and ISC FNIHB Ontario Region.

\section{Results}

\section{Survey Results}

A total of 22 nurses from ISC FNIHB Ontario Region participated in the survey (see Table 1). The majority of the participants worked as a nurse for $15+$ years $(73 \%)$ and are registered nurses $(77 \%)$. Over half of the nurses have worked with FNIM people for over 10+ years (59\%) and currently, almost half (45\%) of nurses serve large First Nations communities (population size 1,000 - 4,999 people).

Table 1.

Demographics of the ISC FNIHB Ontario Region Sioux Lookout Zone Nurses in Charge

\begin{tabular}{|c|c|c|}
\hline \multirow[t]{2}{*}{ Characteristic } & \multicolumn{2}{|c|}{ Sample size } \\
\hline & $n$ & $\%$ \\
\hline \multicolumn{3}{|l|}{ Age range } \\
\hline$<35$ & 2 & $9 \%$ \\
\hline $35-44$ & 4 & $18 \%$ \\
\hline $45-54$ & 9 & $41 \%$ \\
\hline $55-64$ & 5 & $23 \%$ \\
\hline $65+$ & 2 & $9 \%$ \\
\hline \multicolumn{3}{|c|}{ Number of years worked as a nurse } \\
\hline $5-9$ years & 4 & $18 \%$ \\
\hline 10-14 years & 2 & $9 \%$ \\
\hline $15-19$ years & 7 & $32 \%$ \\
\hline $20+$ years & 9 & $41 \%$ \\
\hline \multicolumn{3}{|c|}{ Type of nursing degree } \\
\hline Registered Nurse & 17 & $77 \%$ \\
\hline
\end{tabular}




\begin{tabular}{|l|l|l|}
\hline Nurse Practitioner & 4 & $18 \%$ \\
\hline No Response & 1 & $5 \%$ \\
\hline $\begin{array}{l}\text { Number of years as a nurse working } \\
\text { with FNIM people? }\end{array}$ & & \\
\hline 0-4 years & & \\
\hline 5-9 years & 3 & $14 \%$ \\
\hline $10-14$ years & 6 & $27 \%$ \\
\hline $15-19$ years & 9 & $41 \%$ \\
\hline $20+$ years & 2 & $9 \%$ \\
\hline
\end{tabular}

The majority of the nurses $(86 \%)$ mentioned that the IRCS courses met their expectations. While almost all of the nurses had knowledge of FNIM people before taking the IRCS courses (96\%), 95\% of the nurses found the course content relevant to their career or practice and the majority of the nurses (72\%) found that the program content enhanced their knowledge of FNIM people (see Figure 1). Most of the nurses (82\%) said they will/have applied the learning in their practice (see Figure 2), however, a small percentage (14\%) felt as though the IRCS courses did not add value. Seventy-three percent of the respondents felt that the courses will also help the nurses better understand and work with First Nations patients. All of the nurses (100\%) agreed that the IRCS courses would be of benefit to new nurses and $91 \%$ of the nurses felt that others, beyond nurses, should take the IRCS courses. While $81 \%$ of the nurses found the online learning system to be a good way to deliver the information (see Figure 3 ), half of the nurses (50\%) thought that the online only was the best method for delivering the content and $45 \%$ of the nurses thought that the content should be delivered through a combination of online and in-person facilitation. Key findings of the survey related to the evaluation are displayed as a visual depiction of the results.

The program content enhanced my knowledge of FNIM people

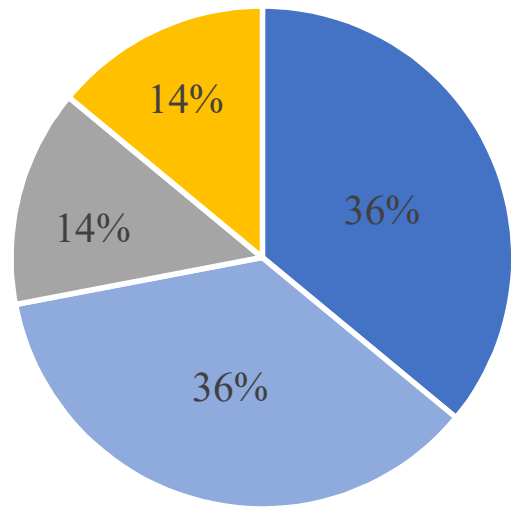

- Strongly agree

- Somewhat agree

- Neither agree nor disagree

- Somewhat disagree

Figure 1. Likert question from the survey 'this program content enhanced my knowledge of FNIM people' 
Applying what was learned in course to my career or practice

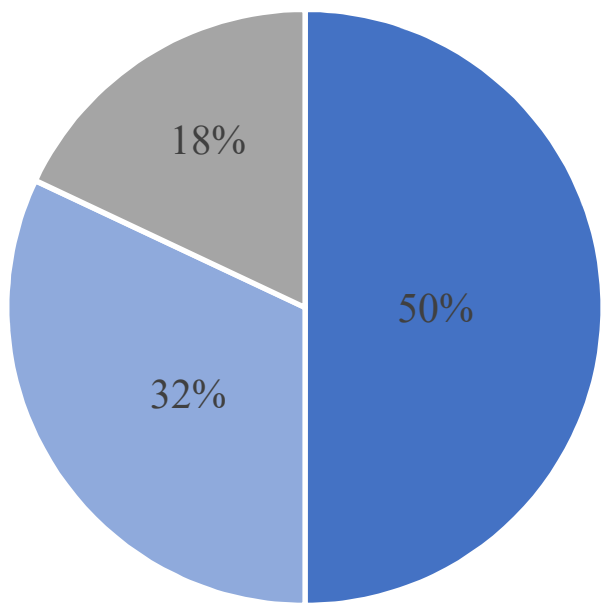

- Strongly agree

- Somewhat agree

- Neither agree nor disagree

Figure 2. Likert question from the survey 'I have/will apply what I learned in this course to my career or practice'

Was the online learning system a good way to deliver the information?

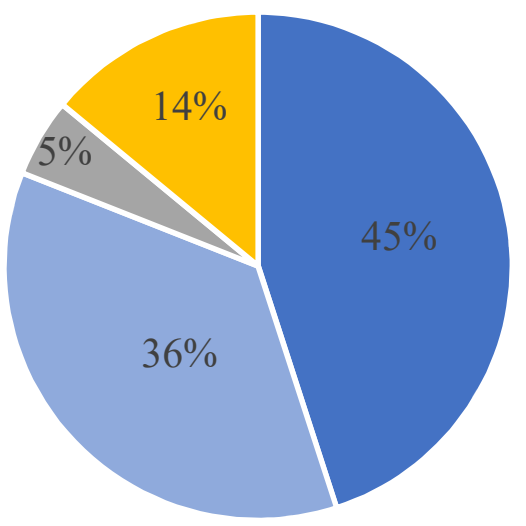

- Strongly agree

- Somewhat agree

- Neither agree nor disagree

- Somewhat disagree

Figure 3. Likert question from the survey 'Do you think the online learning system was a good way to deliver the information?' 


\section{Focus Group Results}

There were a total of eight nurses who participated in the focus group discussion and although the nurses who participated in the focus group also completed the survey portion of the evaluation, demographic information was not collected during the focus groups.

Over the course of an hour, several key themes emerged from the focus group, including: reinforced knowledge, initial resistance, time for completion of the courses, course content and eLearning system specifics, delivery method, and value in the eLearning tool. Each of the key themes had no less than three discussion points, and some themes had an upwards of 10 discussion points. Overall, the discussion was positive towards the IRCS courses as a tool for providing knowledge on Indigenous history, culture and the health landscape to improve health outcomes and person-centred care. Many of the nurses expressed how the IRCS courses reinforced their knowledge of FNIM people.

We work in holistic care, looking at the individual, the family, the whole community, so it is good to take courses that focus on the same issues and reinforce things that we have learned on the job.

The majority of the nurses felt that the content in the IRCS courses were beneficial and enhanced their knowledge of FNIM people. Many of the nurses also felt that the information was clearly delivered and relevant, however some felt that certain parts of the content in the courses were not relevant to their work, such as courses related to the cancer strategy, and courses with cancer screening information.

The cancer strategy, cancer screening modules and all the numbers [statistics] are tedious and feel more like work. They feel disconnected from the rest of the modules.

There was lots of discussion around the delivery method of the cultural competency courses; some nurses liked that the courses were online and self-paced, whereas others thought it would be helpful to first take the courses online and then have an in-person facilitated session. Some participants thought that regardless of the delivery method, it was difficult to find time to take the courses and learn about cultural safety, especially when working full time and having clinic hours. Nonetheless, all of the participants agreed that it would be beneficial for new nurses first starting their career or for nurses who are working with FNIM people for the first time, to take the courses early on.

Having it [IRCS courses] self-paced was helpful, better to have it online and completed at your own time; good for the work environment of nurses.

Some of the nurses shared that they initially felt that they did not need to take the courses because either they were Indigenous or they have worked with FNIM people for many years; however, 
after completing the courses, those participants agreed that they had learned a lot of things related to cultural safety that they did not already know.

If I'm First Nations why do I need to take these courses? But I took them anyways

because they can be applied to other cultures. In the end I learned a lot of things

that I didn't know about my own culture.

There was also consensus that the nurses found value in the learning tool. Many felt that the IRCS courses provided them with knowledge and information that will help to empower them when working with First Nations communities specifically. Some thought that the IRCS courses were a good starting point, but that more needs to be done to ensure that life long learning in cultural humility is embedded into core competencies of the profession.

All healthcare providers that work in the communities should have basic

knowledge, and the IRCS courses give you this basic knowledge.

The IRCS courses don't provide all of the answers, but it's a start. It takes years to be able to gain cultural competency.

\section{Limitations}

The participants in this study were Nurses in Charge who are employed ISC and predominately work with First Nations communities. Given that their primary population is related to the content of the IRCS courses, there is already a certain level of knowledge that the nurses would have compared to the average Canadian, therefore the impact of the courses may not be as great compared to a healthcare worker that does not primarily work with First Nations or Indigenous peoples.

Although the survey and focus group occurred at a Nurse in Charge workshop meeting in Sioux Lookout, with all of the Sioux Lookout Zone nurses in attendance, the sample size was too small to make any inferences. It would not have been possible to conduct power calculations before conducting the study, as we surveyed the maximum amount of Nurses in Charge and could not have sampled more people. There was also less of a spread in the data for the survey responses, due to sample size and the Likert scale questions containing five possible options for response from "strongly agree" to "strongly disagree".

\section{Discussion}

Since the Truth and Reconciliation Commission of Canada released the final report in 2015, cultural competency training has been a large area of focus for many non-Indigenous organizations, institutions, and Canadians (O’Sullivan, 2013). Medical schools and hospitals in Canada are working 
towards implementing the TRC Calls to Action that are relevant to their institutions and professions, recognizing that Canada's healthcare leaders have a role to play in addressing the widespread racism that exists in the healthcare system and contributes to health gaps we continue to see (HealthcareCAN, 2018).

Canadian nursing schools are also embedding cultural competency training into their curriculum from an early onset, recognizing that nurses play a critical role in providing healthcare in northern Canada regions for First Nations and Inuit communities. Nurses are often the main primary care providers in geographically remote communities and thus it is important that cultural competency is a main part of their training and that more Indigenous nurses are recruited into nursing programs (Aboriginal Nurses Association of Canada, 2009).

Cultural competency training is one element in addressing the TRC Calls to Action to provide skills-based training in cultural competency, conflict resolution, human rights, and anti-racism, however it is not the only solution in improving racism in healthcare. Another TRC Call to Action is:

\section{"23. We call upon all levels of government to:}

i. Increase the number of Aboriginal professionals working in the health-care field.

ii. Ensure the retention of Aboriginal health-care providers in Aboriginal communities.

iii. Provide cultural competency training for all healthcare professionals. (TRC, 2015,p.322-323)

It is crucial that medical, nursing and other healthcare professions also put efforts into the recruitment and retention of Indigenous healthcare professionals. Research has demonstrated that nursing schools are more likely to recruit and retain Indigenous nurses when there is a positive environment that has cultural safety in the curriculum and an institution that supports cultural identity for Indigenous students (Thurston \& Mashford-Pringle, 2015). Therefore, it is important that institutions and non-Indigenous organizations recognize the importance of a two-fold approach to address racism in the healthcare system that includes Indigenous cultural competency training to create an environment that supports diversity and inclusion of Indigenous healthcare professionals.

\section{Conclusion}

More research in the field of evaluation will help to understand the impact of the IRCS courses with other healthcare professions, such as physicians and specialists, who may not have received cultural competency training as part of their undergraduate medical education. It will also be important to 
evaluate the impact of the IRCS courses with medical and nursing students who are receiving this cultural competency training as part of their curriculum.

The evaluation of the IRCS courses as a cultural competency training tool demonstrated that free, online, module formatted courses were appropriate and relevant for ISC nurses, that the courses increased their knowledge about FNIM people, and the nurses have/will apply what they learned in their practice. There has been an increasing movement for regions and organizations in Canada to complete cultural competency training. Our evaluation demonstrated that free, online, module formatted courses were successful at meeting learning objectives.

\section{References}

Aboriginal Nurses Association of Canada. (2009). Cultural competency and cultural safety in nursing education. Retrieved from https://www.cna-aiic.ca/ /media/cna/page-content/pdfen/first_nations_framework_e.pdf

Allan, B. \& Smylie, J. (2015). First Peoples, second class treatment: The role of racism in the health and well-being of Indigenous peoples in Canada. Toronto, ON: the Wellesley Institute. Retrieved from http://www.wellesleyinstitute.com/wp-content/uploads/2015/02/Report-First-PeoplesSecond-Class-Treatment-Final.pdf

Brascoupé, S. \& Waters, C. (2009). Cultural safety exploring the applicability of the concept of cultural safety to Aboriginal health and community wellness. Journal of Aboriginal Health, 5(2), 6-41. https://doi.org/10.3138/ijih.v5i2.28981

Canada Health Act, Revised Statutes of Canada (1985, c. C-6). Retrieved from the Justice Laws website: https://laws-lois.justice.gc.ca/eng/acts/C-6/page-1.html\#h-1

Cancer Care Ontario. (2015). Aboriginal Cancer Strategy III 2015 - 2019. Toronto. Retrieved from https://www.cancercareontario.ca/sites/ccocancercare/files/assets/CCOAboriginalStrategy3.pdf

Government of Canada. (2018). Nursing careers. Retrieved from the Government of Canada Indigenous Services website: https://www.canada.ca/en/indigenous-services-canada/services/nursingcareers/apply-now-nursing-jobs.html\#s2

HealthcareCAN. (2018). HealthcareCAN releases wise practices to address the health-related Truth and Reconciliation Commission (TRC) calls to action. Retrieved from http://www.healthcarecan.ca/2018/04/11/healthcarecan-releases-wise-practices-to-address-thehealth-related-truth-and-reconciliation-commission-trc-calls-to-action/

Indian Act, Revised Statutes of Canada (1985, c. I-5). Retrieved from the Justice Laws website: https://laws-lois.justice.gc.ca/eng/acts/I-5/FullText.html 
Jull, J., Giles, A., Minwaashin Lodge, The Aboriginal Women's Support Centre, Boyer, Y., \& Dawn, S. (2015). Cultural adaptation of a shared decision making tool with Aboriginal women: a qualitative study. BMC Medical Informatics and Decision Making, 15(1). https://doi.org/10.1186/s12911-015-0129-7

Mashford-Pringle, A. (2011). How'd we get here from there? American Indians and Aboriginal peoples of Canada health policy. Pimatisiwin: A Journal of Aboriginal and Indigenous Community Health 9(1). Retrieved from http:/www.pimatisiwin.com/online/wpcontent/uploads/2011/08/08Mashford-Pringle.pdf

National Collaborating Centre for Aboriginal Health. (2014). NCCAH Activities Update 2014: Sharing Knowledge, Making a Difference. Retrieved from https://www.ccnsa$\underline{\text { nccah.ca/docs/general/RPT-NCCAHActivitiesUpdate2014-EN.pdf }}$

O’Sullivan, B. (2013). Considering culture in Aboriginal care. CMAJ, 185(1) E27-E28. https://doi.org/10.1503/cmaj.109-4376

Thurston, J.M. \& Mashford-Pringle, A. (2015). Nursing \& indigenous education integration. Journal of Nursing Education and Practice, 5(10). https://doi.org/10.5430/jnep.v5n10p9

Truth and Reconciliation Commission of Canada. (2015). Honouring the Truth, Reconciling for the Future. Retrieved from http://nctr.ca/assets/reports/Final\%20Reports/Executive_Summary_English_Web.pdf 